\title{
Vólvulo gástrico en una paciente nonagenaria: intervención endoscópica y laparoscópica. Reporte de caso
}

\section{Gastric volvulus in a nonagenarian patient: endoscopic and laparoscopic intervention. Case report}

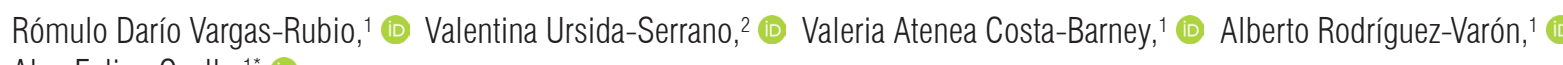
Alan Felipe Ovalle. ${ }^{*}$ (1)

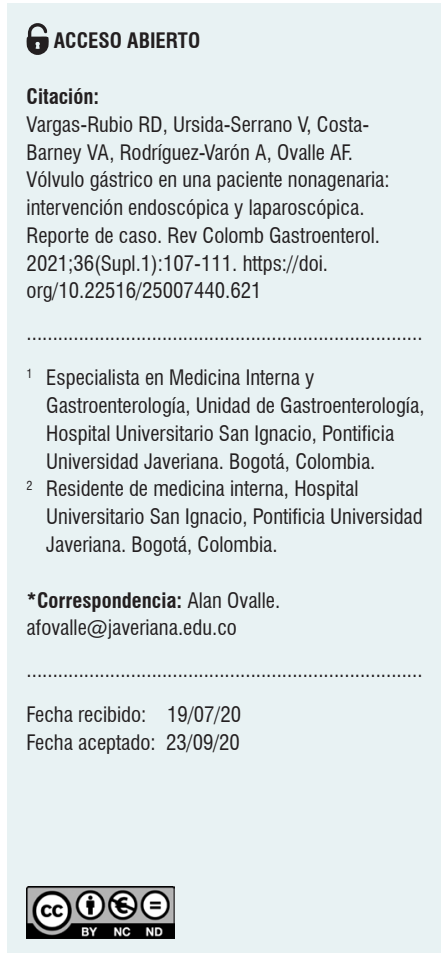

\begin{abstract}
Resumen
El vólvulo gástrico es una entidad rara que puede presentarse de manera aguda o crónica y se acompaña de síntomas inespecíficos. Es fundamental su rápida identificación, ya que tiene alta mortalidad y el tratamiento oportuno de esta patología determina el pronóstico del paciente.

A continuación, presentamos el caso de una paciente femenina de 89 años, que consulta por cuadro clínico de dolor torácico atípico, con documentación en radiografía de tórax de vólvulo gástrico organoaxial, en quien se realiza inicialmente devolvulación endoscópica con éxito. Sin embargo, en las imágenes de control se evidencia recurrencia del vólvulo, por lo cual se realizó manejo quirúrgico con hiatoplastia y funduplicatura tipo Toupet, además de gastrostomía percutánea de fijación, procedimiento realizado sin complicaciones, con los que se logró la devolvulación completa sin recurrencia.
\end{abstract}

\section{Palabras clave \\ Gastropexia, tratamiento endoscópico, vólvulo gástrico.}

\begin{abstract} patient's prognosis.

\section{Keywords}

Gastric volvulus; Endoscopic treatment; Gastropexy.
\end{abstract}

Gastric volvulus is a rare condition that can occur acutely or chronically and is accompanied by nonspecific symptoms. Its rapid identification is critical since it has high mortality rate and timely treatment determines the

The following is the case of an 89-year-old female patient who presented with atypical chest pain, with organoaxial gastric volvulus on chest X-ray, in whom endoscopic devolvulation was initially performed successfully. However, control imaging scans revealed recurrence. Therefore, surgical management included hiatoplasty and Toupet fundoplication, as well as percutaneous fixation gastrostomy, a procedure that was completed without complications and resulted in complete devolvulation without recurrence.

\section{INTRODUCCIÓN}

El vólvulo gástrico es una patología de presentación poco frecuente, que se caracteriza por la rotación anormal del estómago a lo largo de su eje largo o corto, lo que conduce a grados variables de obstrucción (1).

La incidencia alcanza su punto máximo después de la quinta década y los adultos constituyen del $80 \%$ al $90 \%$ de los casos. No se ha informado de ninguna asociación con el sexo o la raza (2).

Su clasificación depende del tiempo de evolución (agudo o crónico), de su etiología (primario o secundario) y de su eje de rotación (organoaxial o mesenteroaxial).

Los síntomas de esta patología no son específicos; sin embargo, cuando se presenta de manera aguda, se asocia a dolor epigástrico, náuseas sin vómito e imposibilidad para 
avanzar la sonda nasogástrica (triada de Borchardt), mientras que si se presenta de manera crónica puede asimilarse a enfermedad ulceropéptica o patología de origen biliar, como colelitiasis (3).

A continuación, se presenta el caso de una paciente nonagenaria, quien consultó a la institución por cuadro clínico de dolor torácico atípico, en quien se documentó vólvulo gástrico organoaxial como hallazgo incidental en la radiografía de tórax de ingreso.

\section{CASO}

Paciente femenina de 89 años con antecedente de osteoporosis y fracturas vertebrales múltiples, quien consulta por cuadro clínico de 4 días de evolución consistente en dolor torácico que aumenta con la inspiración, no irradiado, asociado a disnea en reposo. No manifiesta síntomas gastrointestinales tales como dolor abdominal, náuseas o emesis.

$\mathrm{Al}$ examen físico de ingreso, la paciente está en aceptables condiciones generales, álgida, taquicárdica, diaforética, con dolor a la palpación profunda en epigastrio y sin signos de irritación peritoneal.

Se solicitó radiografía de tórax al ingreso por sospecha de fractura a nivel vertebral, donde se encuentra como hallazgo incidental ascenso de la cámara gástrica en el mediastino posterior, asociado a nivel hidroaéreo, sugestivo de vólvulo gástrico (Figura 1).

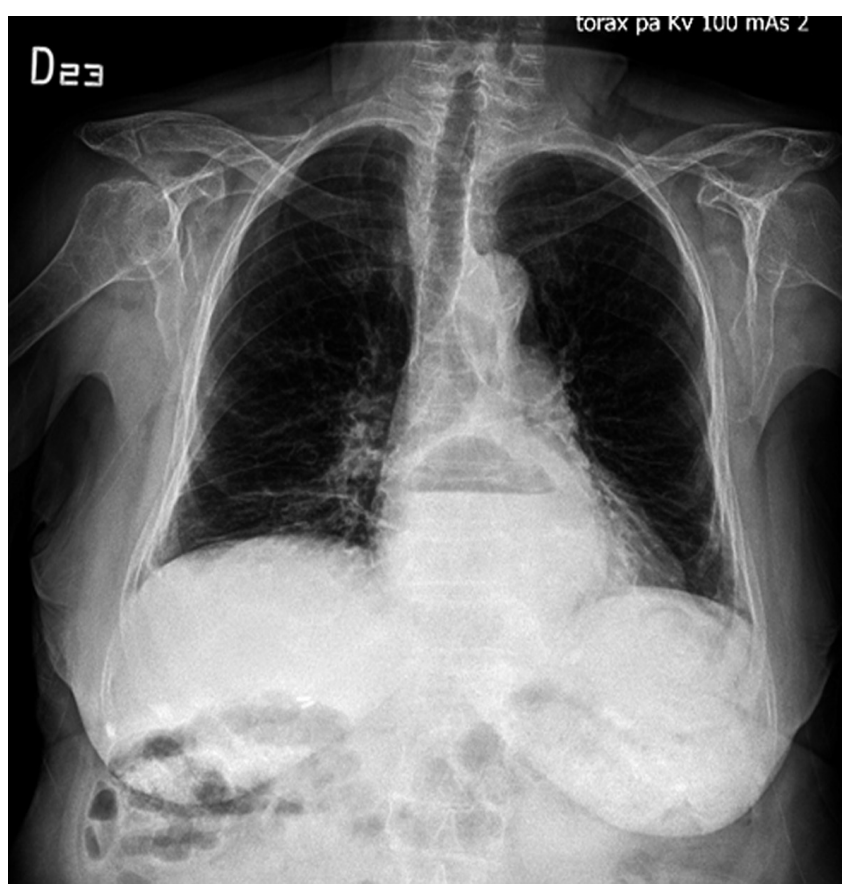

Figura 1. Radiografía de tórax: Se identifica ascenso de la cámara gástrica en el mediastino posterior, con nivel hidroaéreo.
Se inició descompresión gástrica con sonda nasogástrica, manejo de dolor con opioides e hidratación endovenosa con líquidos isotónicos.

Posteriormente se realizó tomografía de abdomen y tórax con contraste, donde se evidencia hernia de la unión gastroesofágica, fondo y unión antropilórica del estómago hacia el tórax, además de una rotación del estómago en su eje largo, que configura vólvulo gástrico organoaxial (Figura 2).

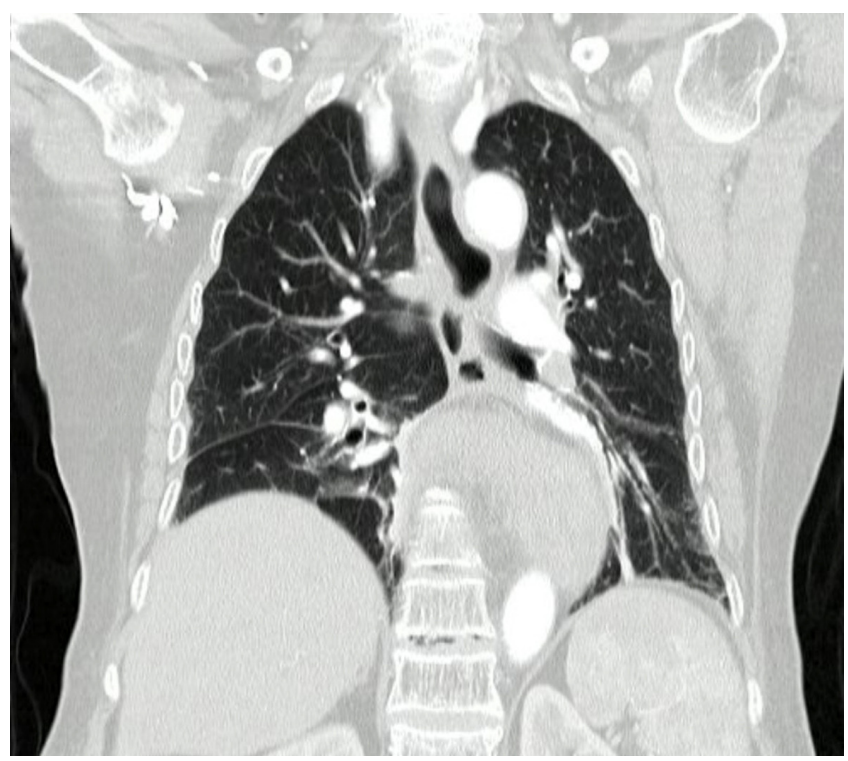

Figura 2. Escanografía de tórax: se observa hernia de la unión gastroesofágica, fondo y unión antropilórica del estómago hacia el tórax, además de rotación del estómago en su eje largo.

Dados los hallazgos encontrados en las imágenes diagnósticas, así como la edad avanzada de la paciente y las comorbilidades que confieren alto riesgo quirúrgico, se decide realizar abordaje endoscópico mediante el que se logra devolvulación gástrica endoscópica, sin complicaciones (Figura 3).

Se realiza nueva radiografía de tórax de control 24 horas después, donde se evidencia recurrencia del vólvulo gástrico.

Ante la recurrencia del vólvulo gástrico posterior al abordaje endoscópico, se decide en conjunto con servicio de cirugía general realizar abordaje quirúrgico, con corrección de hernia hiatal por laparoscopia (hiatoplastia y funduplicatura tipo Toupet) y gastrostomía percutánea de fijación.

Dicho procedimiento fue realizado sin complicaciones, con adecuada evolución de la paciente durante el postoperatorio, tolerancia a la vía oral y mejoría del dolor torácico. La radiografía de tórax de control realizada 72 horas después de procedimiento quirúrgico no muestra recurrencia del vólvulo. 


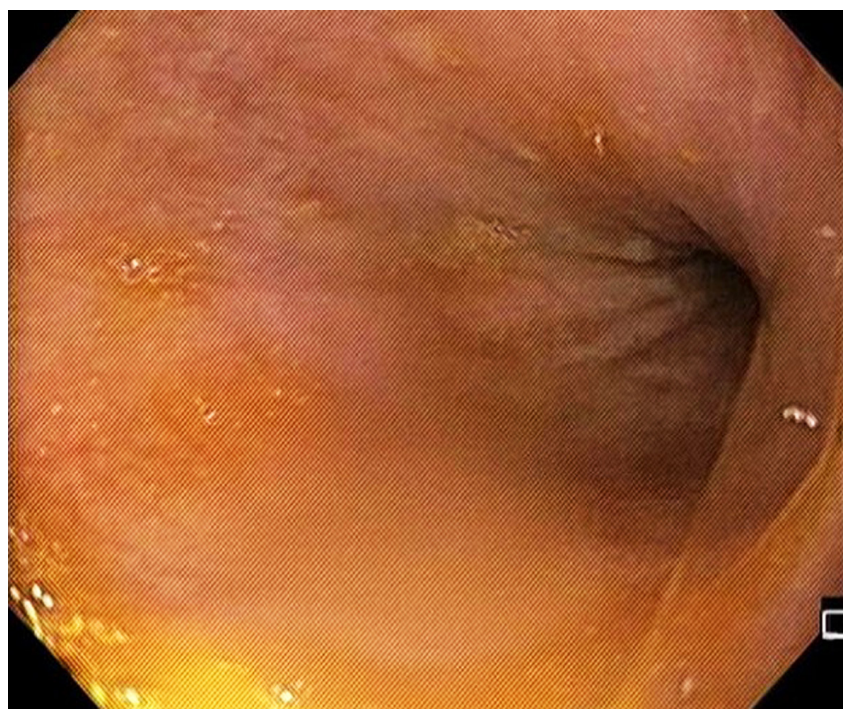

Figura 3. Imagen durante realización de endoscopia de vías digestivas altas y devolvulación endoscópica.

\section{DISCUSIÓN}

El vólvulo gástrico es una patología poco frecuente, que se caracteriza por la rotación anormal del estómago a lo largo de su eje largo o corto, que conduce a grados variables de obstrucción (4).

El diagnóstico y manejo oportuno determina el pronóstico del paciente, dado que puede ocasionar obstrucción, compromiso vascular e incluso estrangulación, que puede evolucionar a necrosis o perforación.

Alrededor del 70\%-80\% de los vólvulos gástricos aparecen en adultos mayores de 50 años, asociados a factores predisponentes como la parálisis del nervio frénico y algunas alteraciones anatómicas del diafragma, órganos abdominales o cifoescoliosis (5).

Existen dos tipos principales: organoaxial, donde se presenta rotación del estómago sobre su eje vertical; y mesenteroaxial, el cual se produce por la rotación sobre el eje horizontal (Figura 4).

El vólvulo gástrico primario (idiopático) se define como un vólvulo debido a las anormalidades de los ligamentos gástricos. La falla de la fijación gástrica puede ocurrir como resultado de agenesia, alargamiento o lesión de los ligamentos gástricos debida a neoplasia, adherencias o deformidad esquelética $(6,7)$.

El vólvulo secundario se define como un vólvulo debido a otras anormalidades anatómicas, como hernia paraesofágica, hernia diafragmática, eventración diafragmática y parálisis del nervio frénico.

El hallazgo clásico del vólvulo gástrico agudo en la radiografía de abdomen simple es la presencia de burbuja gás-
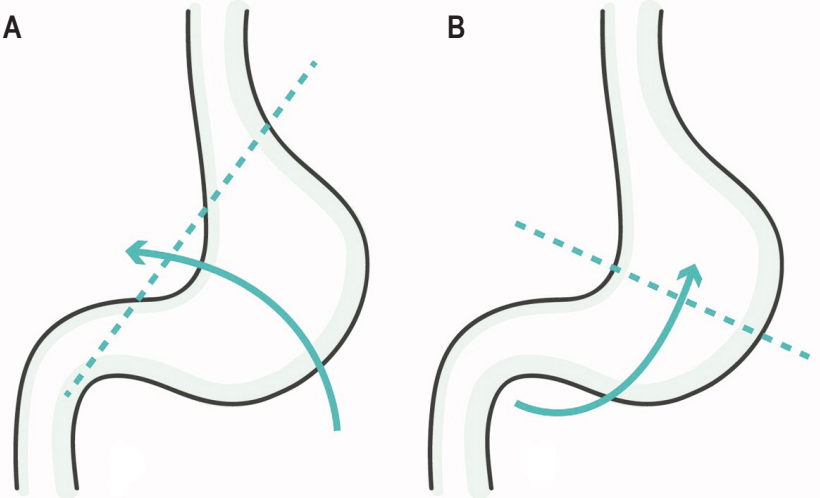

Figura 4. A. Vólvulo organoaxial por rotación del estómago sobre su eje longitudinal. B. Vólvulo mesenteroaxial por rotación del estómago sobre su eje transverso.

trica o nivel hidroaéreo ubicados en la parte superior del abdomen o en el tórax.

En el vólvulo mesenteroaxial, la burbuja gástrica puede ser doble (una en el fundus y otra en el antro), mientras que, en el vólvulo organoaxial, el estómago se observa en posición horizontal y solo se ve una burbuja.

La tomografía axial computarizada tiene la ventaja adicional de mostrar la relación del estómago con las estructuras circundantes e identificar anormalidades anatómicas asociadas al vólvulo gástrico secundario.

El vólvulo agudo, como se presenta en el caso de nuestra paciente, usualmente requiere manejo con descompresión endoscópica, devolvulación endoscópica inicial, gastrostomía endoscópica percutánea, gastropexia y reparación de los defectos estructurales predisponentes, con el fin de prevenir la recurrencia (8). En el estudio realizado por YaoChun Hsu y colaboradores, en donde se realizó el seguimiento a 44 pacientes con diagnóstico de vólvulo gástrico, los cuales fueron manejados conservadoramente ante la presencia de síntomas leves a moderados, con un tiempo de seguimiento de 36 meses y una mediana de edad de 71 años, se evidenció una recurrencia del $64 \%$, sin que ninguno de los pacientes desarrollara complicaciones agudas durante el estudio; sin embargo, un paciente requirió cirugía laparoscópica ante la presencia de síntomas recurrentes con alteración de la calidad de vida $(9,10)$. El tiempo promedio libre de recurrencia fue de 3 meses y los factores asociados se relacionaron con un mayor tiempo de duración entre el inicio de los síntomas y el inicio del diagnóstico (1,6 meses; $p=0,065)$, cirugía torácica y abdominal previas $(p=0,061)$ y adherencias peritoneales $(p=0,015)(11)$. 
De otro lado, se ha descrito que el manejo quirúrgico es superior al manejo con gastrostomía endoscópica percutánea como manejo único, lo cual no previene la recurrencia. Clásicamente, el vólvulo gástrico se ha manejado quirúrgicamente mediante la combinación con técnicas endoscópicas en algunos pacientes, incluida la devolvulación sola o la devolvulación con una o dos gastrostomías percutáneas (12). El abordaje endoscópico está limitado por la obstrucción en la unión gastroesofágica en pacientes con vólvulo organoaxial que impida el paso seguro del gastroscopio y a su vez favorezca la dificultad para la orientación del estómago; además, el manejo endoscópico no aborda la patología diafragmática que se observa en la mayoría de los casos de vólvulo gástrico.

Se han descrito varios métodos quirúrgicos para el manejo del vólvulo gástrico, en los cuales se incluyen la reparación de hernias diafragmáticas, gastropexia, gastrectomía parcial por necrosis, gastrogastrotomía fondo antral (operación de Opolzer) y gastropexia con fijación del ligamento gastrocólico (operación de Tanner), entre otras.

El manejo combinado (quirúrgico con gastrostomía endoscópica percutánea prequirúrgica o intraoperatoria) favorece que el tubo de gastrostomía sirva como ancla principal del estómago a la pared abdominal anterior, lo que permite el reposicionamiento laparoscópico de las suturas de gastropexia, para lograr una posición anatómica óptima del estómago a lo largo de su eje natural, sin que se describa el impacto de la realización de gastrostomía endoscópica previa a la cirugía o durante esta. Es una limitante para su realización prequirúrgica el paso del gastroscopio a través de la unión esofagogástrica o la no devolvulación endoscópica (13).

Se han descrito dos técnicas para realizar la devolvulación vía endoscópica, entre las cuales encontramos la maniobra Alfa-loop, descrita por Tsang en 1995, la cual comprende seis pasos principales, en los cuales se forma un asa en alfa en el extremo proximal del estómago volvulado mediante el avance de la punta del endoscopia por el sitio de la estenosis que produce el vólvulo; posteriormente se lleva la punta del endoscopia al duodeno realizando un torque del endoscopia en el sentido de las manecillas del reloj. También se ha descrito la maniobra endoscópica dual bajo fluoroscopia, en la cual se lleva el gastroscopio de adultos hasta el duodeno, lo cual favorece el desplazamiento del estómago hacia la cavidad abdominal y el reposicionamiento del antro manteniendo su eje, con posterior paso de un gastroscopio pediátrico al lado del gastroscopio de adultos y realización de una gastrostomía percutánea endoscópica para fijación de la pared anterior del estómago a la pared abdominal (14).

En nuestro caso, ante la recurrencia del vólvulo a pesar del manejo endoscópico, y con la intención de realizar la fijación gástrica para evitar una nueva recurrencia, se decide en conjunto con servicio de cirugía general realizar abordaje quirúrgico con hiatoplastia y funduplicatura tipo Toupet, además de gastrostomía percutánea de fijación, con los que se logra la devolvulación completa, sin nueva recurrencia y con una evolución adecuada.

\section{CONCLUSIÓN}

El vólvulo gástrico es una entidad infrecuente, con un espectro de presentación clínica que va desde cuadros crónicos caracterizados por síntomas inespecíficos hasta una presentación clínica aguda, con síntomas de inicio súbito y alto riesgo de mortalidad.

Es importante la sospecha y el diagnóstico oportuno con imágenes radiológicas, las cuales permiten identificar la patología, así como el mecanismo de ocurrencia y las complicaciones asociadas. La endoscopia temprana es necesaria y se requiere tratamiento con gastropexia endoscópica o laparoscópica como una alternativa menos invasiva y viable a un procedimiento quirúrgico más agresivo en algunos pacientes adultos mayores con comorbilidades importantes.

\section{REFERENCIAS}

1. Rashid F, Thangarajah T, Mulvey D, Larvin M, Iftikhar S. A review article on gastric volvulus: a challenge to diagnosis and management. Int J Surg. 2010;8(1):18. https://doi.org/10.1016/j.ijsu.2009.11.002

2. Chau B, Dufel S. Gastric volvulus. Emerg Med J. 2007;24:446. https://doi.org/10.1136/emj.2006.041947

3. Godshall D, Mossallam U, Rosenbaum R. Gastric volvulus: case report and review of the literature. J Emerg Med. $1999 ; 17(5): 837-40$. https://doi.org/10.1016/S0736-4679(99)00092-X
4. Teague WJ, Ackroyd R, Watson DI, Devitt PG. Changing patterns in the management of gastric volvulus over 14 years. Br J Surg. 2000;87:358. https://doi.org/10.1046/j.1365-2168.2000.01385.x

5. Wu MH, Chang YC, Wu CH, Kang S, Kuan J. Acute gastric volvulus: a rare but real surgical emergency. Am J Emerg Med. 2010;28(1):118.e5-118.e7. https://doi.org/10.1016/j.ajem.2009.04.031

6. Shivanand G, Seema S, Srivastava DN, Pande G, Sahni P, Prasad R, et al. Gastric volvulus: acute and chronic presen- 
tation. Clin Imaging. 2003;27(4):265-8.

https://doi.org/10.1016/S0899-7071(02)00549-1

7. Wasselle JA, Norman J. Acute gastric volvulus: pathogenesis, diagnosis, and treatment. Am J Gastroenterol. 1993;88(10):1780-4.

8. Flanagan NM, McAloon J. Gastric volvulus complicating cerebral palsy with kyphoscoliosis. Ulster Med J. 2003;72(2):118-20.

9. Ayala JA, Naik-Mathuria B, Olutoye OO. Delayed presentation of congenital diaphragmatic hernia manifesting as combined-type acute gastric volvulus: a case report and review of the literature. J Pediatr Surg. 2008;43(3):E35-9. https://doi.org/10.1016/j.jpedsurg.2007.11.015

10. McElreath DP, Olden KW, Aduli F. Hiccups: a subtle sign in the clinical diagnosis of gastric volvulus and a review of the literature. Dig Dis Sci. 2008;53:3033-6.

https://doi.org/10.1007/s10620-008-0258-2
11. Hsu YC, Perng CL, Chen CK, Tsai JJ, Lin HJ. Conservative management of chronic gastric volvulus: 44 cases over 5 years. World J Gastroenterol. 2010;16(33):4200-5. https://doi.org/10.3748/wjg.v16.i33.4200

12. Ghosh S, Palmer KR. Double percutaneous endoscopic gastrostomy fixation: an effective treatment for recurrent gastric volvulus. Am J Gastroenterol. 1993;88(8):1271-2.

13. Newman RM, Newman E, Kogan Z, Stien D, Falkenstien D, Gouge TH. A combined laparoscopic and endoscopic approach to acute primary gastric volvulus. J Laparoendosc Advanced Surg Tech. 1997;7(3):177-81. https://doi.org/10.1089/lap.1997.7.177

14. Jamil LH, Huang BL, Kunkel DC, Jayaraman V, Soffer EE. Successful gastric volvulus reduction and gastropexy using a dual endoscope technique. Case Rep Med. 2014;136381. https://doi.org/10.1155/2014/136381 